\title{
A humanized anti-IGF-1R monoclonal antibody (R1507) and/or metformin enhance gemcitabine-induced apoptosis in pancreatic cancer cells
}

\author{
TAKAKO KAWANAMI ${ }^{1}$, SOICHI TAKIGUCHI ${ }^{1}$, NORIKO IKEDA $^{2}$ and AKIHIRO FUNAKOSHI ${ }^{3}$ \\ ${ }^{1}$ Institute for Clinical Research, National Kyushu Cancer Center; ${ }^{2}$ Department of Internal \\ Medicine and Bioregulatory Science, Graduate School of Medical Sciences, Kyushu University; \\ ${ }^{3}$ Fukuoka Sanno Hospital and International University of Health and Welfare, Fukuoka, Japan
}

Received August 26, 2011; Accepted October 10, 2011

DOI: 10.3892/or.2011.1597

\begin{abstract}
Pancreatic cancer is a disease with a dismal prognosis and treatment options are limited. This study investigated the interaction of gemcitabine with R1507 and/ or metformin and the induction of an inhibitor of apoptosis protein by this combination. Pancreatic cancer cells were treated with gemcitabine, R1507 and metformin alone or in combination. The effects of treatments were evaluated for cell proliferation, apoptosis, and the expression of genes related to inhibition of apoptosis and chemotherapy resistance. Combination of gemcitabine with R1507 and/or metformin additively interacted with the inhibition of cell proliferation in human pancreatic ductal adenocarcinoma cell lines, SUIT-2 and MIAPaCa- 2 with differential gemcitabine resistance, and assessment of apoptosis demonstrated that drug associations increased the apoptotic index in both cell lines. Treatment with gemcitabine induced the expression of survivin and XIAP in both cell lines, indicating the induction of chemoresistance. In conclusion, these data demonstrate that the combination of gemcitabine with R1507 and/or metformin has an additive effect in pancreatic cancer cell lines with differential sensitivity to gemcitabine; however, gemcitabine may induce chemotherapy resistance.
\end{abstract}

\section{Introduction}

Pancreatic cancer is the fourth most common cause of cancer death in men and women in the US, with 5-year survival for all stages of disease $<5 \%$ (1). Pancreatic cancer has no clear early warning signs or symptoms and is usually silent until

Correspondence to: Dr Soichi Takiguchi, Institute for Clinical Research, National Kyushu Cancer Center, 3-1-1 Notame, Minami-ku, Fukuoka 811-1395, Japan

E-mail: sctakigu@nk-cc.go.jp

Key words: pancreatic cancer, gemcitabine resistance, R1507, metformin, apoptosis, chemoresistance the disease is well advanced. Patients have a median survival of 4-8 months after diagnosis due in part to the advanced stage of disease by the time it is discovered and treatment is begun. It is therefore necessary to discover new therapies or therapeutic combinations to make significant impact on this deadly disease. The anti-metabolite agent gemcitabine is currently being employed to treat pancreatic cancer (2). While gemcitabine has shown a significant benefit in clinical applications, its ability to more than modestly affect pancreatic cancer is limited. It has been speculated that combinatory treatments using gemcitabine and other chemotherapeutics could improve survival rates (3).

The involvement of the insulin-like growth factor/insulinlike growth factor 1 receptor (IGF/IGF-1R) axis in tumorigenesis makes it an attractive target for anticancer therapeutics. R1507, a fully human $\mathrm{IgG1}$ type monoclonal antibody directed against human IGF-1R, has been developed to antagonize IGF-1R signaling. R1507 binds the extracellular domain of IGF-1R with high affinity and selectivity, resulting in the displacement of bound IGF-1 and loss of receptor protein on the cell surface due to intracellular internalization and degradation. R1507 inhibits tumor proliferation of the prostate, lung, breast, pancreas, and sarcoma cell lines at concentrations $<10 \mathrm{nmol} / 1$ and delays tumor growth in xenograft models (4-6). Recently we found pancreatic cancer cell lines expressed IGF-1R moderately and R1507 bound to the IGF-1R on membrane. Furthermore, R1507 showed significant survival effect in nude mouse intraperitoneal dissemination model with Suit-2 pancreatic cancer cell line (7). Metformin, a drug used in the treatment of diabetes for decades, decreases the growth of pancreatic ductal adenocarcinoma (PDAC) cell lines and could be a potential candidate in novel treatment strategies for PDAC patients (8). Metformin is an activator of AMP-activated protein kinase (AMPK) which inhibits protein synthesis and gluconeogenesis during cellular stress. The main downstream effect of AMPK activation is the inhibition of mammalian target of rapamycin (mTOR), a downstream effector of growth factor signaling. mTOR is frequently activated in malignant cells and is associated with resistance to anticancer drugs (9).

Survivin, a member of the inhibitor of apoptosis protein (IAP) family, has previously been shown to be a prognostic 
marker for pancreatic cancer patients (10-12) and has also been implicated in cancer cell chemotherapy resistance (13). Many recent reports have demonstrated that inhibiting survivin expression by antisense oligonucleotides (14), dominant negative mutation $(15,16)$, and ribozyme (17) can reduce cancer cell radio- and chemoresistance and may be important to resensitize these tumors to therapy. XIAP, a unique and bestcharacterized member of the IAP family, has been identified as a central regulator of caspase-dependent apoptosis. Whether the activation of apoptosis is initiated by events that perturb the mitochondria (via caspase-9) or progress directly from cell surface receptors (via caspase-8), the ability of XIAP to inhibit the downstream executioner caspases- 3 and -7 makes it a potent and broad inhibitor of cell death and an important target for therapy (18).

In the present study, we evaluated the combined effect of gemcitabine with R1507 and/or metformin on pancreatic cancer cell lines with differential sensitivity to gemcitabine and examined the induction of survivin and XIAP.

\section{Materials and methods}

Cell culture. SUIT-2 (19), MIAPaCa-2 (20), BxPC-3, AsPC-1, Panc-1, H-48-N and TCC-PAN2 were derived from a human PDAC. All human PDAC cell lines were cultured in RPMI1640 medium (Invitrogen, Carlsbad, CA, USA) supplemented with $10 \% \mathrm{FBS}$ and $50 \mathrm{U} / \mathrm{ml}$ penicillin and $50 \mu \mathrm{g} / \mathrm{ml}$ streptomycin in a humidified atmosphere under $5 \% \mathrm{CO}_{2}$ at $37^{\circ} \mathrm{C}$. Immortalized human pancreatic ductal cell line HPDE6E6E7-c7 (H6c7) is a generous gift from Dr Ming-Sound Tsao (University Health Network, Toronto, ON, Canada) $(21,22)$. It was cultured in HuMedia-KG2 medium (Kurabo, Osaka, Japan) in a humidified atmosphere under $5 \% \mathrm{CO}_{2}$ at $37^{\circ} \mathrm{C}$.

Drug sensitivity test. Human PDAC were seeded into 96-well plates (MIAPaCa-2, 2.5x10 cells/50 $\mu$ l; SUIT-2, $1.25 \times 10^{3}$ cells/ $50 \mu \mathrm{l})$ and allowed to attach for $24 \mathrm{~h}$. Cells were treated with a) gemcitabine $(0.01-100 \mu \mathrm{M})$ (Toronto Research Chemicals, North York, ON, Canada); b) R1507 (0.1-300 nM) (kindly provided by Roche Diagnostics GmbH, Mannheim, Germany); c) metformin (0.5-100 mM) (Sigma-Aldrich, St. Louis, MO, USA) for $24 \mathrm{~h}$ and $48 \mathrm{~h}$ in $50 \mu \mathrm{l}$ medium. At the end of drug exposure, $20 \mu \mathrm{l}$ 3-(4,5-dimethylthiazol-2-yl)-5-(3-carboxymethoxyphenyl)2-(4-sulfophenyl)-2H-tetrazolium (MTS) reagent (Promega, Madison, WI, USA) was added and cells were incubated in humidified $5 \% \mathrm{CO}_{2}$ atmosphere. After $2 \mathrm{~h}$, spectrophotometric readings were taken for each sample according to the manufacturer's instructions. Cell growth inhibition was expressed as a percentage of the absorbance of control cultures measured at $490 \mathrm{~nm}$ with a microplate reader. The $50 \%$ inhibitory concentration of cell growth $\left(\mathrm{IC}_{50}\right)$ was calculated by a sigmoidal dose-response curve (GraphPad PRISM, San Diego, CA, USA). Drug interactions among gemcitabine, R1507, metformin or their combinations were assessed at a fixed concentration for $48 \mathrm{~h}$ exposure. The final concentration of each drug was set at $0.5 \times \mathrm{IC}_{50}$. The $\mathrm{IC}_{50}$ values after $48 \mathrm{~h}$ exposure were applied for gemcitabine and metformin treatment, whereas the $\mathrm{IC}_{50}$ values after $24 \mathrm{~h}$ exposure were applied for R1507 treatment. Accordingly, R1507 was added twice at 0 and $24 \mathrm{~h}$ in the course of $48 \mathrm{~h}$ exposure.

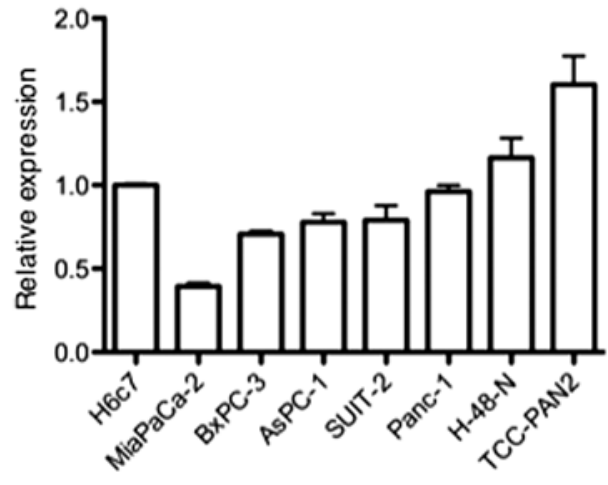

Figure 1. Relative expression of IGF-1R in PDAC cell lines. Total RNA was extracted from PDAC cell lines and an immortalized human pancreatic ductal cell line H6c7 cultured under normal conditions. Quantitative RT-PCR analysis was performed. Expression levels of IGF-1R were normalized with $\beta$-actin. The expression of $\mathrm{H} 6 \mathrm{c} 7$ was set as one. $\mathrm{N}=3$ for each experiment. Results are shown as the average \pm SD

Analysis of apoptosis. Human pancreatic cancer cell lines were cultured in slide chambers to no more than 50-60\% confluence and treated with gemcitabine, R1507, metformin or their combinations, as described in the 'drug sensitivity test'. After 48-h treatment, apoptosis was assessed using the in situ cell death detection kit (Roche Applied Science, Mannheim, Germany), following the manufacturer's recommendations. Briefly, cells were washed with PBS once, fixed with $4 \%$ buffered paraformaldehyde for $1 \mathrm{~h}$, washed again with PBS, and permeabilized on ice with $0.1 \%$ Triton X-100 in $0.1 \%$ sodium citrate for $2 \mathrm{~min}$. Slides were rinsed twice with PBS and then incubated for $60 \mathrm{~min}$ at $37^{\circ} \mathrm{C}$ with terminal deoxynucleotidyl transferase enzyme in reaction buffer. The slides were rinsed three times with PBS and mounted with PermaFluor (Thermo Fisher Scientific, Waltham, MA, USA). Samples were analyzed by fluorescence microscopy. TUNEL-positive nuclei were detected by a bright color in condensed or ruptured nuclei. The rate of apoptosis was calculated as the ratio of the number of apoptotic cells to the total number of cells (both apoptotic and non-apoptotic cells).

Quantitative real-time PCR. Total RNA was extracted from pancreas cancer cell lines treated with anti-cancer drugs using an RNeasy RNA isolation kit (Qiagen, Venlo, The Netherlands) according to the manufacturer's instructions. cDNA was transcribed using a $20-\mu 1$ volume with the ThermoScript RT-PCR System and a random hexamer (Invitrogen) according to the manufacturer's instructions. All PCR reactions were carried out in a final volume of $25 \mu \mathrm{l}$ and were performed using the ABI PRISM 7000 Sequence Detection System (Applied Biosystems, Foster City, CA, USA) according to the manufacturer's protocol. Sequence-specific primers are quoted from internet site 'PrimerBank' (23) and summarized in Table I. The reaction mix consisted of SYBR Premix Ex Taq (2x) (Takara Bio, Otsu, Japan) $12.5 \mu 1$, ROX Reference Dye (x50) (Takara) $0.5 \mu \mathrm{l}, 0.2 \mu \mathrm{M}$ of each specific forward and reverse primer, and $9 \mu \mathrm{l}$ diluted cDNA (equivalent to $0.03-2.85 \mathrm{ng}$ total RNA). Amplifications were performed under standard conditions (10 sec at $95^{\circ} \mathrm{C}$ followed by 40 cycles of $5 \mathrm{sec}$ at $95^{\circ} \mathrm{C}$ and $31 \mathrm{sec}$ at $60^{\circ} \mathrm{C}$ ). The number of PCR cycles needed to reach the 
Table I. Gene-specific primer pairs for real-time RT-PCR.

\begin{tabular}{llc}
\hline Human gene & \multicolumn{1}{c}{ Primer sequence $\left(5^{\prime}-3^{\prime}\right)$} & Length (bp) \\
\hline IGF-1R & Sense: AGGATATTGGGCTTTACAACCTG & 145 \\
& Antisense: GGCTTATTCCCCACAATGTAGTT & \\
Survivin & Sense: AGGACCACCGCATCTCTACAT & 118 \\
XIAP & Antisense: AAGTCTGGCTCGTTCTCAGTG & 101 \\
$\beta$-actin & Sense: ATAGTGCCACGCAGTCTACAA & 250 \\
& Antisense: AGATGGCCTGTCTAAGGCAAA & \\
\hline
\end{tabular}

A

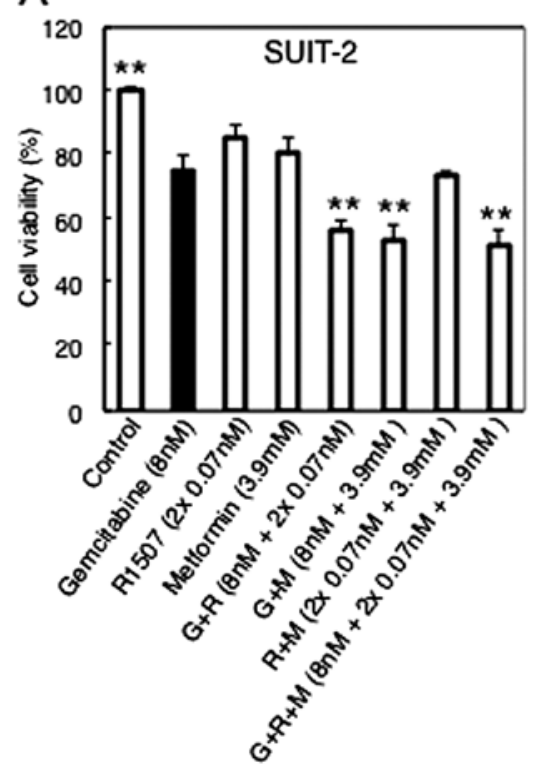

$\mathrm{B}$

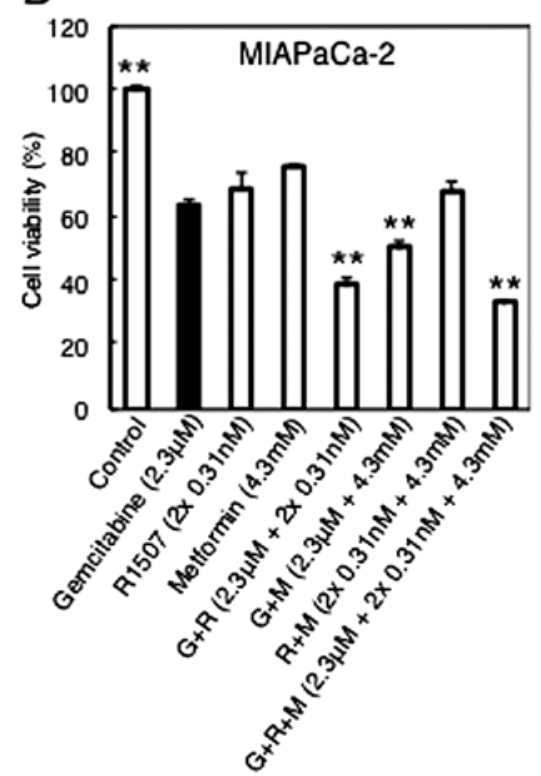

Figure 2. Combination treatment of gemcitabine with R1507 and/or metformin on the viability of SUIT-2 (A) and MIAPaCa-2 (B) cells. Cells were treated with gemcitabine, R1507, metformin or their combinations at a fixed concentration. Cell growth inhibition was expressed as a percentage of the absorbance of control cultures measured at $490 \mathrm{~nm}$ with a microplate reader. $\mathrm{N}=3$ for each experiment. Results are shown as the average $\pm \mathrm{SD}$. ${ }^{*} \mathrm{p}<0.05,^{* *} \mathrm{p}<0.01$ vs. gemcitabine treatment.

fluorescence threshold was determined in triplicate for each cDNA, averaged, and then normalized to a reference gene $(\beta$-actin). A standard curve was generated with serial 3-fold dilutions of representative cDNA. For all assays tested, the PCR reaction was linear over the range studied (20-40 cycles of amplification). All RT-PCR reactions gave a single band when analyzed by gel electrophoresis.

Statistical analysis. Results are reported as the average \pm SD. Group comparisons were performed using one-way analysis of variance (ANOVA) followed by Tukey-Kramer's multiple comparison test. Differences were considered significant at $\mathrm{p}<0.05$.

\section{Results}

Relative expression of IGF-1R, the target of the humanized monoclonal antibody (R1507), was investigated in PDAC cell lines. An immortalized human pancreatic ductal cell line $\mathrm{H} 6 \mathrm{c} 7$ was used as a control. The results indicate that all PDAC cell lines express IGF-1R at levels comparable to that of $\mathrm{H6c7}$ (Fig. 1).

The cytotoxicity of gemcitabine, R1507 and metformin against pancreatic cancer cell lines was examined by the MTS assay and the values of $\mathrm{IC}_{50}$ were calculated. Gemcitabine, R1507 and metformin inhibited the growth of the SUIT-2 and MIAPaCa-2 cell lines in a concentration-dependent manner, and the $\mathrm{IC}_{50}$ values are summarized in Table II. Our results indicate that $\mathrm{MIAPaCa}-2$ is 300 -fold more resistant to gemcitabine than SUIT-2. While the $\mathrm{IC}_{50}$ of R1507 and metformin were comparable between the two cell lines. Next the drug interaction of gemcitabine with R1507 and/or metformin was assessed at a fixed concentration for $48 \mathrm{~h}$ treatment. As a single treatment of R1507 was more potent at $24 \mathrm{~h}$ exposure than at $48 \mathrm{~h}$ exposure, we planned to add R1507 at 0 and $24 \mathrm{~h}$ referring to the values of $\mathrm{IC}_{50}$ for $24 \mathrm{~h}$ exposure. 
Table II. In vitro cytotoxicity of gemcitabine, R1507 and metformin on human pancreatic cancer cells, SUIT-2 and MIAPaCa-2.

\begin{tabular}{lcccc}
\hline Cell name & Exposure & \multicolumn{2}{c}{$\mathrm{IC}_{50}$} \\
\cline { 3 - 5 } & $24 \mathrm{~h}$ & $\begin{array}{c}\text { Gemcitabine } \\
(\mu \mathrm{M})\end{array}$ & $\begin{array}{c}\mathrm{R} 1507 \\
(\mathrm{nM})\end{array}$ & $\begin{array}{c}\text { Metformin } \\
(\mathrm{mM})\end{array}$ \\
\hline SUIT-2 & $48 \mathrm{~h}$ & 0.0023 & 0.13 & 5.6 \\
& $24 \mathrm{~h}$ & 0.0015 & $>100$ & 7.8 \\
MIAPaCa-2 & $48 \mathrm{~h}$ & 0.71 & 0.61 & 10.9 \\
& & 0.45 & $>100$ & 8.5 \\
\hline
\end{tabular}
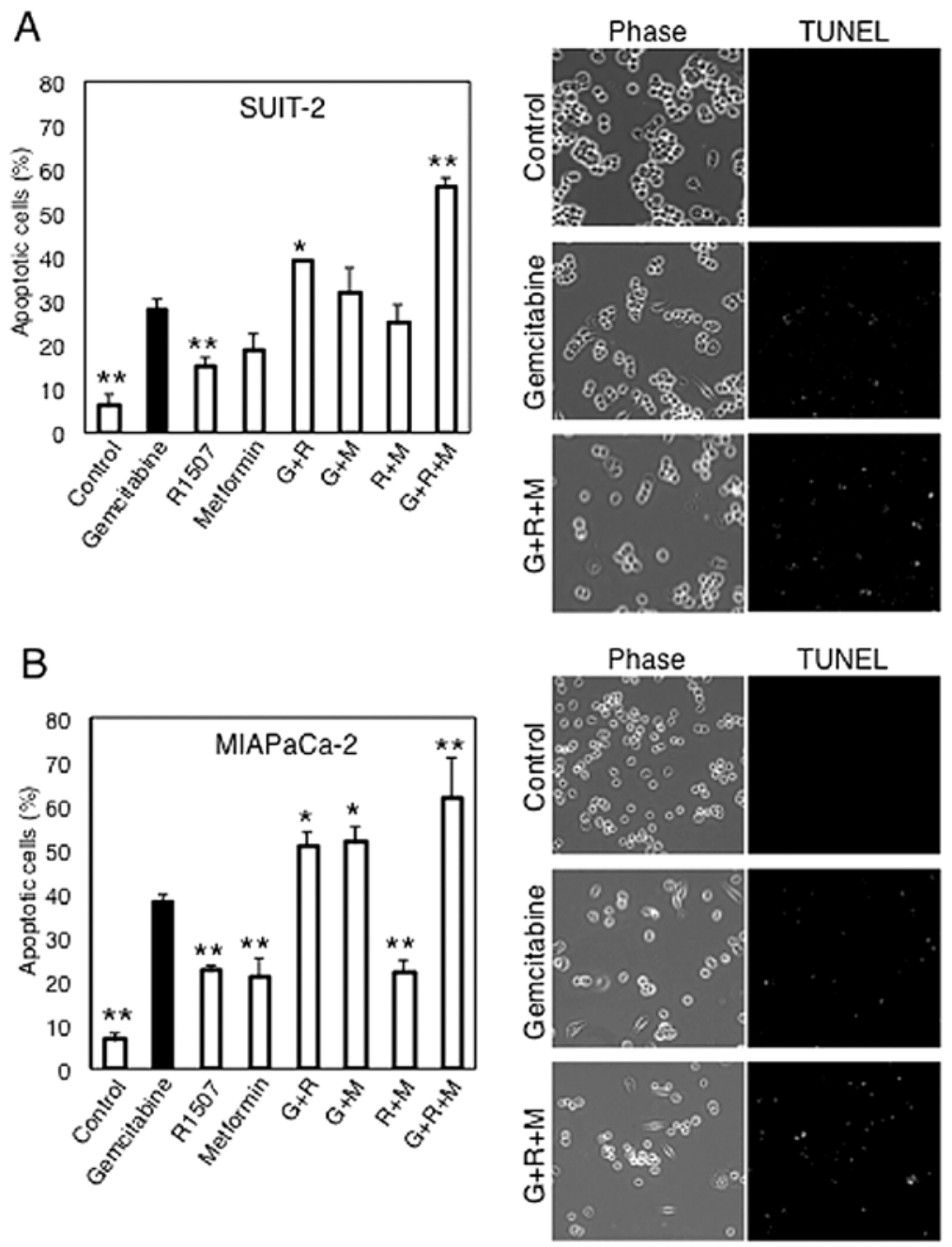

Figure 3. Combination treatment of gemcitabine with R1507 and/or metformin on the induction of apoptosis in SUIT-2 (A) and MIAPaCa-2 (B) cells. Cells were treated with gemcitabine, R1507, metformin or their combinations at a fixed concentration, as shown in Fig. 1. The rate of apoptosis was calculated as the ratio of the number of apoptotic cells to the total number of cells. $\mathrm{N}=3$ for each experiment. Results are shown as the average $\pm \mathrm{SD}$. ${ }^{*} \mathrm{p}<0.05,{ }^{* *} \mathrm{p}<0.01 \mathrm{vs}$. gemcitabine treatment (left). Representative microscopic photos of control, gemcitabine-treated and gemcitabine/ R1507/ metformin-treated PDAC cell lines (right).

Consequently, the combinations of gemcitabine with R1507 and/or metformin were more potent than gemcitabine, R1507 or metformin alone in both cell lines, indicating an additive effect, while there was no additive effect with the combination of R1507 and metformin (Fig. 2).

To explore the reason for the additive cytotoxic effects of gemcitabine, R1507 and/or metformin, the induction of apoptosis was analyzed by TUNEL assay. Upon exposure to gemcitabine, R1507, metformin, and their combinations, pancreatic cancer cells presented typical apoptotic morphology with cell shrinkage, nuclear condensation and fragmentation, and cellular rupture into debris. The occurrence of apoptosis was significantly higher in gemcitabine-treated cells than R1507and/or metformin-treated cells (Fig. 3). R1507 and metformin 

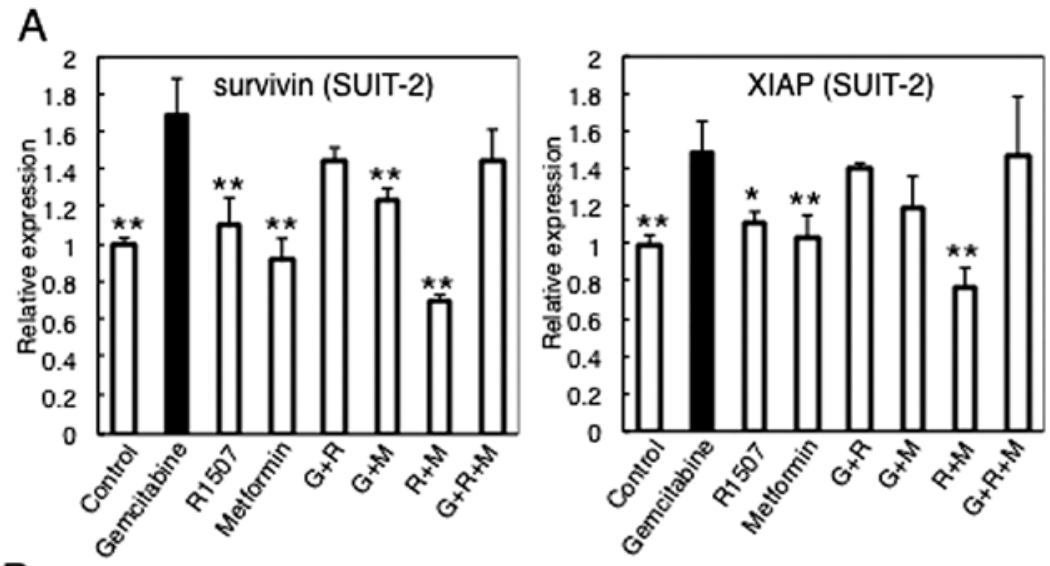

B
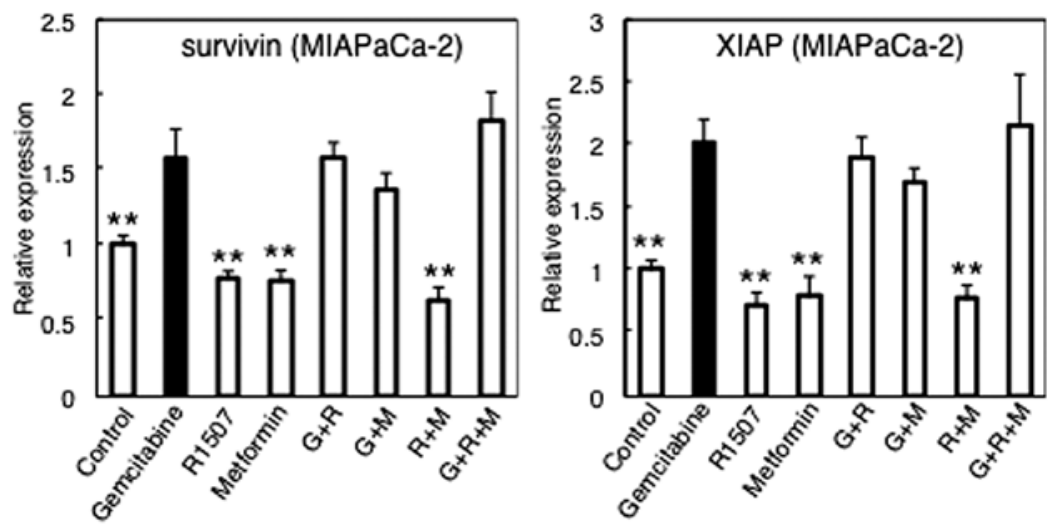

Figure 4. Quantitative RT-PCR analysis of survivin and XIAP mRNAs in SUIT-2 (A) and MIAPaCa-2 (B) cells. Cells were treated with gemcitabine, R1507, metformin or their combinations at a fixed concentration, as shown in Fig. 1. Expression levels of survivin and XIAP were normalized with $\beta$-actin. $\mathrm{N}=4$ for each experiment. Results are shown as the average $\pm \mathrm{SD}$. ${ }^{*} \mathrm{p}<0.05,{ }^{* *} \mathrm{p}<0.01$ vs. gemcitabine treatment.

had additive effects on gemcitabine-induced apoptosis in both cell lines (Fig. 3).

We investigated the expression levels of the IAP family, survivin and XIAP during chemotherapy treatments by quantitative real-time PCR. Treatment with gemcitabine induced the expression of survivin and XIAP significantly in both cell lines, while treatment with R1507 and metformin did not affect the expression of survivin and XIAP in either cell line (Fig. 4).

\section{Discussion}

Pancreatic cancer remains one of the most difficult malignancies to treat. Even patients who are eligible for curative surgical resection gain only modest benefits. Furthermore, the lack of effective chemotherapeutic and targeted agents highlights the urgent need to better understand pancreatic cancer biology to guide the identification and development of novel treatment strategies. The nucleoside analog gemcitabine provided encouraging results in both antitumor effects and its impact on parameters of clinical benefit for patients with pancreatic cancer such as, decreased pain severity, decreased requirement for opioid analgesics, increased appetite and weight gain (24). In direct comparison on locally advanced pancreatic cancer and metastatic pancreatic cancer, gemcitabine treatment resulted in a 5.56 month overall survival compared to a 4.41 month overall survival using 5-FU (25). As a result, gemcitabine has become widely accepted for unresectable pancreatic cancer. However, the combination treatment using gemcitabine with other agents has not been successful in increasing the overall survival. These disappointing results call for novel combination therapies to improve the survival outcome of pancreatic cancer patients.

The aim of the current study was to evaluate the combined effect of gemcitabine with R1507 and/or metformin on pancreatic cancer cell lines. The growth inhibitory effects of R1507 and metformin may be mediated through inhibition of the IGF-1R pathway and the downstream mTOR pathway (26). Whereas gemcitabine is a nucleoside analogue metabolized to triphosphate moiety by deoxycytidine kinase and is incorporated into the nascent DNA and blocks DNA replication (27). We combined the drugs with two different modes of action for the treatment of two pancreatic cancer cell lines with differential sensitivity to gemcitabine. Consequently, the combination of gemcitabine with R1507 and/or metformin was more potent than gemcitabine, R1507 or metformin alone, indicating an additive effect in both cell lines. While there was no additive effect with the combination of R1507 and metformin, which suggest the majority of the growth inhibitory effects of R1507 and metformin are mediated through inhibition of the IGF-1R pathway and the downstream mTOR pathway. Supporting the cytotoxicity assay, the R1507 and/or metformin have additive effects on gemcitabine-induced apoptosis in both cell lines. However, our data suggest that treatment with gemcitabine may induce chemotherapy resistance in both cell lines through 
the induction of survivin and XIAP, while treatment with R1507 and metformin does not. Survivin has been pursued as a therapeutic target, given its role in chemoresistance and its preferential expression in malignant cells over normal cells $(28,29)$. Both antisense and chemical XIAP inhibitors were developed as therapeutic agents for the treatment of malignancy (30). Their combination with IAP inhibitors might be effective against chemoresistance caused by gemcitabine treatment. In conclusion, the combination of gemcitabine with R1507 and/or metformin has an additive effect in pancreatic cancer cells with differential sensitivity to gemcitabine; however, gemcitabine may induce chemotherapy resistance.

\section{References}

1. Kleeff J, Michalski C, Friess $\mathrm{H}$ and Buchler MW: Pancreatic cancer: from bench to 5-year survival. Pancreas 33: 111-118, 2006.

2. Ueno H, Kiyosawa K and Kaniwa N: Pharmacogenomics of gemcitabine: can genetic studies lead to tailor-made therapy? $\mathrm{Br}$ J Cancer 97: 145-151, 2007.

3. Reni M, Cereda S and Galli L: PEFG (cisplatin, epirubicin, 5-fluorouracil, gemcitabine) for patients with advanced pancreatic cancer: the ghost regimen. Cancer Lett 256: 25-28, 2007.

4. Kurzrock R, Patnaik A, Aisner J, et al: A phase I study of weekly R1507, a human monoclonal antibody insulin-like growth factor-I receptor antagonist, in patients with advanced solid tumors. Clin Cancer Res 16: 2458-2465, 2010.

5. Gong Y, Yao E, Shen R, et al: High expression levels of total IGF-1R and sensitivity of NSCLC cells in vitro to an anti-IGF-1R antibody (R1507). PLoS One 4: e7273, 2009.

6. Kolb EA, Kamara D, Zhang W, et al: R1507, a fully human monoclonal antibody targeting IGF-1R, is effective alone and in combination with rapamycin in inhibiting growth of osteosarcoma xenografts. Pediatr Blood Cancer 55: 67-75, 2010.

7. Funakoshi A, Kawanami T and Takiguchi S: Anti-tumor effect of the insulin-like growth factor (IGF)-1 receptor (IGF-1R) on human pancreatic cancer cells in both in vitro and in vivo studies. Suizo (In press).

8. Kisfalvi K, Eibl G, Sinnett-Smith J and Rozengurt E: Metformin disrupts crosstalk between $G$ protein-coupled receptor and insulin receptor signaling systems and inhibits pancreatic cancer growth. Cancer Res 69: 6539-6545, 2009.

9. Jalving M, Gietema JA, Lefrandt JD, et al: Metformin: taking away the candy for cancer? Eur J Cancer 46: 2369-2380, 2010.

10. Sarela AI, Verbeke CS, Ramsdale J, Davies CL, Markham AF and Guillou PJ: Expression of survivin, a novel inhibitor of apoptosis and cell cycle regulatory protein, in pancreatic adenocarcinoma. Br J Cancer 86: 886-892, 2002.

11. Kami K, Doi R, Koizumi M, et al: Survivin expression is a prognostic marker in pancreatic cancer patients. Surgery 136: 443-448, 2004.

12. Satoh K, Kaneko K, Hirota M, Masamune A, Satoh A and Shimosegawa T: Expression of survivin is correlated with cancer cell apoptosis and is involved in the development of human pancreatic duct cell tumors. Cancer 92: 271-278, 2001.
13. Li F: Survivin study: what is the next wave? J Cell Physiol 197: 8-29, 2003.

14. Kim KW, Mutter RW, Willey CD, et al: Inhibition of survivin and aurora B kinase sensitizes mesothelioma cells by enhancing mitotic arrests. Int J Radiat Oncol Biol Phys 67: 1519-1525, 2007.

15. Asanuma K, Kobayashi D, Furuya D, Tsuji N, Yagihashi A and Watanabe N: A role for survivin in radioresistance of pancreatic cancer cells. Jpn J Cancer Res 93: 1057-1062, 2002.

16. Mesri M, Wall NR, Li J, Kim RW and Altieri DC: Cancer gene therapy using a survivin mutant adenovirus. J Clin Invest 108: 981-990, 2001.

17. Pennati M, Binda M, Colella G, et al: Radiosensitization of human melanoma cells by ribozyme-mediated inhibition of survivin expression. J Invest Dermatol 120: 648-654, 2003.

18. Mufti AR, Burstein E and Duckett CS: XIAP: cell death regulation meets copper homeostasis. Arch Biochem Biophys 463: 168-174, 2007.

19. Iwamura T, Katsuki $\mathrm{T}$ and Ide K: Establishment and characterization of a human pancreatic cancer cell line (SUIT-2) producing carcinoembryonic antigen and carbohydrate antigen 19-9. Jpn J Cancer Res 78: 54-62, 1987.

20. Yunis AA, Arimura GK and Russin DJ: Human pancreatic carcinoma (MIA PaCa-2) in continuous culture: sensitivity to asparaginase. Int J Cancer 19: 128-135, 1977.

21. Furukawa T, Duguid WP, Rosenberg L, Viallet J, Galloway DA and Tsao MS: Long term culture and immortalization of epithelial cells from normal adult human pancreatic ducts by transfection with the E6E7 gene of human papilloma virus 16. Am J Pathol 148: 1763-1770, 1996.

22. Ouyang H, Mou LJ, Luk C, Liu N, Squire J and Tsao MS: Immortal human pancreatic duct epithelial cell lines with near normal genotype and phenotype. Am J Pathol 157: 1623-1631, 2000.

23. Spandidos A, Wang X, Wang H and Seed B: PrimerBank: a resource of human and mouse PCR primer pairs for gene expression detection and quantification. Nucleic Acids Res 38: D792-D799, 2010.

24. Burris HA III, Moore MJ, Andersen J, et al: Improvements in survival and clinical benefit with gemcitabine as first-line therapy for patients with advanced pancreas cancer: a randomized trial. J Clin Oncol 15: 2403-2413, 1997.

25. Park JK, Ryu JK, Lee JK, et al: Gemcitabine chemotherapy versus 5-fluorouracil-based concurrent chemoradiotherapy in locally advanced unresectable pancreatic cancer. Pancreas 33: 397-402, 2006.

26. Pollak M: Insulin and insulin-like growth factor signalling in neoplasia. Nat Rev Cancer 8: 915-928, 2008.

27. Hidalgo M: Pancreatic cancer. N Engl J Med 362: 1605-1617, 2010.

28. Nakahara T, Takeuchi M, Kinoyama I, et al: YM155, a novel small-molecule survivin suppressant, induces regression of established human hormone-refractory prostate tumor xenografts. Cancer Res 67: 8014-8021, 2007.

29. Carrasco RA, Stamm NB, Marcusson E, Sandusky G, Iversen P and Patel BK: Antisense inhibition of survivin expression as a cancer therapeutic. Mol Cancer Ther 10: 221-232, 2011.

30. Schimmer AD, Dalili S, Batey RA and Riedl SJ: Targeting XIAP for the treatment of malignancy. Cell Death Differ 13: 179-188, 2006. 